\title{
Prediction of the Critical Line of a Binary Mixture: Evaluation of the Interaction Parameters
}

\author{
B. E. Eaton* \\ Department of Chemical Engineering, University of Colorado, Boulder, CO 80307 \\ and \\ J. Stecki** and P. Wielopolski** \\ Institułe of Physical Chemistry, Polish Academy of Sciences, Warsaw, Poland \\ and \\ H. J. M. Hanley $†$ \\ Department of Chemical Engineering, University of Colorado, Boulder, CO 80307 \\ and \\ National Bureau of Standards, † Boulder, CO 80307
}

March 23, 1981

\begin{abstract}
The critical line of the binary mixture methane-ethane is calculated via the extended corresponding states Van der Waals one fluid theory. The Gibbs free energy criticality criteria are solved numerically. The numerical derivatives are compared with the exact analytical results derived previously for the special case of the shape factors of the extended corresponding states set equal to unity. Binary interaction parameters are adjusted to give a best fit of the critical line to experimental data. These interaction parameters are then used to evaluate vapor liquid equilibrium data away from the critical region. It appears that a fit of the critical line is not sufficient to obtain binary interaction parameters of general applicability. Optimization of the critical point predictions for the pure components is also discussed.
\end{abstract}

Key words: Binary interaction parameters; criticality criteria; extended corresponding states; gas-liquid critical line; one fluid theory; Van der Waals theory; VLE prediction.

\section{Introduction}

The prediction of phase equilibria is both a classical problem of the theory of liquids and a problem of engineering concern. Today the chemical and fuel industries have to increase productivity and conservation and transfer to new

\footnotetext{
-Supported by the National Science Foundation, Grant No. HES 7419548, SMI 7610647.

*"Supported in part by the Maria Curie Sklodowska Fund, Grant No. NBS-196, established by contributions of the U.S. and Polish governments.

†Supported in part by the Office of Standard Reference Data, NBS.

††Thermophysical Properties Division, National Engineering Laboratory.
}

feedstocks; phase equilibria is a major factor. But it is well known that the prediction, even the correlation, of the properties of the appropriate systems can be exceptionally difficult if the results are required to any reasonable accuracy. Prediction techniques are needed especially because the number of possible systems makes measurement an overwhelming task. Prediction requires an understanding of theory but, unfortunately, theory cannot yet always handle adequately the complex systems encountered: the gap between a systematic practical theory and reality is large. One technique, however, has been applied successfully to simple systems and does show promise in that the assumptions can 
be identified clearly. This method is extended corresponding states. Here we apply it to a system of methane and ethane. A specific objective is to calculate the gas/liquid critical line and to observe the effect of the binary interaction parameters on the calculation. It is then interesting to see how these parameters, optimized for the critical line, represent vapor liquid equilibrium (VLE) data.

The critical line in a binary mixture may be calculated by solving the equations

$$
\left(\frac{\partial^{2} \widetilde{\mathrm{G}}}{\partial x^{2}}\right)_{T, p}=0 ;\left(\frac{\partial^{3} \widetilde{\mathrm{G}}}{\partial x^{3}}\right)_{T, p}=0
$$

for a temperature $(T)$ and pressure $(p)$ with the mole fraction $(x)$ specified. $\vec{G}$ is the molar configurational Gibbs free energy of the mixture. In this work these second and third order derivatives were evaluated numerically, but have been compared with the analytical results of Wielopolski $[1]^{1}$ in the special case when the extended corresponding states shape factors are unity. The accuracy of the approach has thus been evaluated.

The system methane/ethane was selected for comparison with experiment since the VLE data have been evaluated for thermodynamic consistency by Hiza, et al. [2]. The procedure is quite general, however, and we have applied it to several mixtures. Variations have been reported extensively by Watson and Rowlinson [3], Gunning and Rowlinson [4], Teja and Rowlinson [5], Mollerup and Rowlinson [6], and Mollerup [7, 8]. The overall objective is to develop a general technique for calculating the critical line of a binary mixture and to see if the binary interaction parameters can be reliably evaluated by adjusting them to give the best least squares fit of the critical line data.

\section{Corresponding states and equations}

The basic postulate of the theory used here-the Van der Waals one fluid theory-is that if the components $\alpha(\alpha=$ $1, n$ where $n$ is the total number of species) of a mixture separately obey classical corresponding states, then their mixture will also obey corresponding states as if it were a single substance. The components can be represented by selected parameters, e.g., critical temperature $\left(T_{\alpha}^{c}\right)$ and critical molar volume $\left(V_{\alpha}^{c}\right)$, and the hypothetical equivalent substance, designed by subscript $x$, can be characterized by some suitable composition dependent averaged parameters $T_{s}^{c}$ and $V_{x}$. The method then assumes that the properties of a pure substance at $p$ and $T$, or $V$ and $T$, can be evaluated with respect to those of a reference fluid, designated by subscript $o$, via

$$
T_{o}=T / f_{a \alpha, o} \text { and } V_{o}=V / h_{\alpha \alpha, o}
$$

where the scaling ratios $h$ and $f$ are defined respectively by

$$
h_{\alpha \alpha, o}=V_{\alpha}^{c} / V_{o}^{c} \text { and } f_{\alpha \alpha, o}=T_{\alpha}^{c} / T_{o}^{c}
$$

For a mixture the most natural definition of $h_{x}$ and $f_{x}$ follows from the work of Henderson and Leonard [9] to give the Van der Waals one fluid mixing rules:

$$
\begin{aligned}
h_{x, 0} f_{x, 0} & =\sum_{\alpha} \sum_{\beta} x_{\alpha} x_{\beta} f_{\alpha \beta, o} h_{\alpha \beta, 0} \\
h_{x, o} & =\sum_{\alpha} \sum_{\beta} x_{\alpha} x_{\beta} h_{\alpha \beta, o}
\end{aligned}
$$

The cross coefficients $f_{\alpha \beta, o}$ and $h_{\alpha \beta, o}$ are left unspecified until further combination rules are defined, e.g.,

$$
\begin{gathered}
f_{\alpha \beta, 0}=\xi_{\alpha \beta, 0}\left(f_{\alpha \alpha, 0} f_{\beta \beta, 0}\right)^{1 / 2} \\
h_{\alpha \beta, \rho}=\eta_{\alpha \beta, o}\left[1 / 2\left(h_{\alpha \alpha, o}\right)^{1 / 3}+1 / 2\left(h_{\beta \beta, o}\right)^{1 / 3}\right]^{3}
\end{gathered}
$$

where $\xi_{\alpha \beta, 0}$ and $\eta_{\alpha \beta, 0}$ are the binary interaction coefficients which, although formally close to unity, can play a major role in the calculation of phase equilibria.

\section{One Fluid Mixture Equations:}

The properties of a mixture can be evaluated in terms of the reference substance and the ratios of eq (2). The basic equations are:

Compressibility factor, $Z$

$$
\begin{aligned}
Z(T, V, x) & \equiv Z_{x}(T, V, x) \\
& =Z_{o}\left(T / f_{x, o}, V / h_{x, o}\right)
\end{aligned}
$$

Molar configurational Helmholtz free energy, $\tilde{A}$

$$
\widetilde{A}(V, T, x) \equiv \widetilde{A}_{x}(V, T, x)+R T \sum_{\alpha} x_{\alpha} \ln x_{\alpha}
$$

where

$$
\widetilde{A}_{x}(V, T, x)=f_{x, 0} \tilde{A}_{o}\left(V / h_{x, 0}, T / f_{x, 0}\right)-R T \ln h_{x, 0}
$$

or the molar configurational Gibbs free energy, $\widetilde{G}$

$$
\widetilde{G}(p, T, x) \equiv \widetilde{G}_{x}(p, T, x)+R T \sum_{\alpha} x_{\alpha} \ln x_{\alpha}
$$

where

$$
\widetilde{G}_{x}(p, T, x)=f_{x, 0} \widetilde{G}_{0}\left(p h_{x, 0} / f_{x, 0}, T / f_{x, 0}\right)-R T \ln h_{x, 0}
$$

'Numbers in brackets refer to the literature references at the end of this paper. 
The symbol $\sim$ refers to the molar quantity. Equations (7)-(12) which define the properties of an $n$-component mixture, can also be used for pure component properties if all subscript $x$ 's are replaced with $\alpha$ 's.

\section{VLE Equations:}

For pure component VLE, equating the molar Gibbs free energy of each phase results in the following expression:

$$
\begin{aligned}
& {\left[\tilde{A}_{o}^{\text {Res }} / R T_{0}-\ln Z_{o}-1+Z_{0}\right]_{v a p}=} \\
& {\left[\tilde{A}_{o}^{R o s} / R T_{0}-\ln Z_{o}-1+Z_{0}\right]_{1 / q}} \\
& \widetilde{A}_{0}^{r e s} \equiv \widetilde{A}\left(T / f_{\alpha \alpha, o}, V / h_{\alpha \alpha, o}\right)-\widetilde{A}_{p g}\left(T / f_{\alpha \alpha, o}, V / h_{\alpha \alpha, o}\right)
\end{aligned}
$$

In eq (13) superscript $R e s$ refers to the residual value defined by eq (14) with $\widetilde{A}_{p g}$ the value of the equivalent perfect gas. Equation (13) is expressed in terms of the residual Helmholtz free energy rather than Gibbs since the reference equation of state has $T$ and $V$ (not $T$ and $p$ ) as the independent variables.

For mixture VLE one can calculate the $K$-value for, say, species $\alpha$ at $T$ and $p$ :

$$
K_{\alpha}=y_{\alpha} / x_{\alpha}
$$

where one can derive

$$
R T \ln K_{\alpha}=\left(\mu_{\alpha}^{R e s}\right)_{L_{i q}}-\left(\mu_{\alpha}^{R_{\alpha}}\right)_{V a p}
$$

with $\mu^{\text {Res }}$ the residual chemical potential. Further manipulations give $\mu^{R e s}$ in terms of $\widetilde{G}$ and, for a binary mixture,

$$
\mu_{\alpha}^{R e s}=\widetilde{G}_{x}-R T x_{\beta}\left(\frac{\partial\left(\widetilde{G}_{x} / R T\right)}{\partial x_{\beta}}\right)_{r, p}-R T \ln \frac{p}{k T}
$$

where

$$
\frac{\widetilde{G}_{x}}{R T}=\frac{\widetilde{A}_{o}^{\text {Ros }}}{R T_{\mathrm{o}}}-\ln Z_{o}-1+Z_{o}+\ln \frac{p}{k T}
$$

\section{Critical Criteria:}

The conditions for a critical point at $T, p$ for a mixture are

$$
\left(\partial^{2} \widetilde{G} / \partial x^{2}\right)_{T, \rho}=\left(\partial^{3} \widetilde{G} / \partial x^{3}\right)_{T, p}=0 .
$$

Substitution of the one fluid equations gives

$$
\left(\partial^{2}\left(\widetilde{G}_{x} / R T\right) / \partial x_{\alpha}^{2}\right)_{T, p}+\frac{1}{x_{\alpha} x_{\beta}}=0
$$

and

$$
\left(\partial^{3}\left(\tilde{G}_{x} / R T\right) / \partial x_{\alpha}^{3}\right)_{T, p}+\frac{x_{\alpha}-x_{\beta}}{\left(x_{\alpha} x_{\beta}\right)^{2}}=0
$$

which can thus be evaluated using eq (18).

The above equations and others have been discussed in full and derived by Rowlinson and Watson [3], by Eaton [10] and by other authors so it has been sufficient to be very brief. The equations form the basics of the evaluation of phase equilibria for a pure fluid or mixture, given the reference equation of state and the reference $G_{o}$ or $A_{o}$.

\section{Extended Corresponding States:}

In general, since classical corresponding states is not obeyed, equations (8) and (10) or (8) and (12) are not satisfied with the scaling ratios of eq (2). It is possible, however, to define a corresponding states so that eqs (8) and (10) are satisfied exactly. To do this we define shape factors $\theta$ and $\phi$ so that (for a pure, for example)

$$
T_{o}=T\left(\frac{T_{o}^{\mathrm{c}}}{T_{\alpha}^{\mathrm{c}}}\right) \frac{1}{\theta_{\alpha \alpha, o}} \quad ; \quad V_{o}=V\left(\frac{V_{o}^{\mathrm{c}}}{V_{\alpha}^{c}}\right) \frac{1}{\phi_{\alpha \alpha, o}}
$$

hence the ratios $f$ and $h$ become

$$
f_{\alpha \alpha, o}=\left(\frac{T_{\alpha}^{\mathrm{c}}}{T_{\mathrm{o}}^{\mathrm{c}}}\right) \theta_{\alpha \alpha, \rho} \quad ; \quad h_{\alpha \sigma, o}=\left(\frac{V_{\mathrm{a}}^{\mathrm{c}}}{V_{\mathrm{o}}^{\mathrm{c}}}\right) \phi_{\alpha \alpha, \rho} .
$$

The point about this redefinition, i.e., the basis of extended corresponding states theory, is that the corresponding states equations can be used formally with the provision that the scaling ratios are given by eq (23). It should be stressed that the ratios could be solved for either a pure or a mixture via eqs (8) and (10) but to do this would require a complete description of the fluids in question: essentially an impossibility. It is convenient to have some generalized analytical relation for $\theta$ and $\phi$. Leach and Leland proposed the following [11]:

$$
\begin{array}{r}
\theta_{\alpha \alpha, o}\left(T_{\alpha}^{*}, V_{\alpha}^{*}, \omega_{\alpha}\right)=1+\left(\omega_{\alpha}-\omega_{o}\right) F\left(T_{\alpha}^{*}, V_{\alpha}^{*}\right) \\
\phi_{\alpha \alpha, o}\left(T_{\alpha}^{*}, V_{\alpha}^{*}, \omega_{\alpha}\right)=\left[1+\left(\omega_{\alpha}-\omega_{0}\right) G\left(T_{\alpha}^{*}, V_{\alpha}^{*}\right)\right] \frac{Z_{\alpha}^{c}}{Z_{\alpha}^{c}}
\end{array}
$$

where

$$
F\left(T_{\alpha}^{*}, V_{\alpha}^{*}\right)=a_{1}+b_{1} \ln T_{\alpha}^{*}+\left(c_{1}+d_{1} / T_{\alpha}^{*}\right)\left(V_{\alpha}^{*}-0.5\right)
$$

and

$$
G\left(T_{\alpha}^{*}, V_{\alpha}^{*}\right)=a_{2}\left(V_{\alpha}^{*}+b_{2}\right)+c_{2}\left(V_{\alpha}^{*}+d_{2}\right) \ln T_{\alpha}^{*}
$$

Here $\omega$ is the pitzer acentric factor or some chosen parameter and $a, b, c, d$ are constants: 


$$
\begin{array}{ll}
a_{1}=0.0892, & a_{2}=0.3903 \\
b_{1}=-0.8493, & b_{2}=-1.0177 \\
c_{1}=0.3063, & c_{2}=-0.9462 \\
d_{1}=-0.4506, & d_{2}=-0.7663
\end{array}
$$

The asterisk denotes the value reduced by the critical value. The equations are constrained in that $V_{a}^{*}$ is set equal to 2.0 for all $V_{a}^{*}>2.0$ and to 0.5 for $V_{a}^{*}<0.5: T_{a}^{*}$ is set to 2.0 if $T_{a}^{*}>2.0$.

We [12] have recently tested the Leach-Leland equations for the hydrocarbons $C_{1}-C_{20}$ over an extensive range of experimental conditions and revised coefficients are reported in the reference. We also verified that the original equations were satisfactory for reduced temperatures greater than 0.5 .

\section{Calculation and numerical methods}

The objective is to solve the critical criteria eqs (20) and (21) for the methane/ethane system and in so doing, observe the effects of the interaction parameters $\xi$ and $\eta$ of eqs (5) and (6) on the results. Having these values, we then evaluate some $K$-values for selected temperatures using eqs (15)-(18). We chose methane as the reference fluid, the equation of state for which is the 32 term BWR of McCarty [13]. Critical parameters and Leach-Leland acentric factors for methane and ethane are given in table 1.

TABLE 1. Parameters for Methane and Ethane

\begin{tabular}{llccc}
\hline & $\begin{array}{c}T^{k} \\
(\mathrm{~K})\end{array}$ & $\begin{array}{c}\widetilde{V}^{c} \\
\left(\mathrm{~cm}^{3 / m o l e}\right)\end{array}$ & $\begin{array}{c}p^{e} \\
(\mathrm{Bar})\end{array}$ & $\omega$ \\
\hline $\mathrm{CH}_{4}$ & 190.555 & 97.75 & 44.793 & .005 \\
$\mathrm{C}_{2} \mathrm{H}_{6}$ & 305.33 & 147.06 & 47.448 & .105 \\
\hline
\end{tabular}

Analytical and Numerical Evaluation of the Derivatives:

The numerical techniques used in this work are standard. We use the central difference formulas [14] for which the first two terms in the infinite power series expansions are given here. For the derivatives of a function $f$ evaluated at a point $x$, one has

$$
\begin{gathered}
\left.\frac{d f}{d x}\right)_{x} \approx \frac{f_{1}-f_{-1}}{2 h}-\frac{f_{2}-2 f_{1}+2 f_{-1}-f_{-2}}{12 h} \\
\left.\frac{d^{2} f}{d x^{2}}\right)_{x}=\frac{f_{1}-2 f_{0}+f_{-1}}{h^{2}}-\frac{f_{2}-4 f_{1}+6 f_{0}-4 f_{-1}+f_{-2}}{12 h^{2}} \\
\left.\frac{\partial^{3} f}{\partial x^{3}}\right)_{x} \approx \frac{f_{2}-2 f_{1}+2 f_{-1}-f_{-2}}{2 h^{3}-8 h^{3}}- \\
\frac{f_{3}-4 f_{2}+5 f_{1}-5 f_{-1}+4 f_{-2}-f_{-3}}{8 h^{3}}
\end{gathered}
$$

where

$$
f_{0}=f(x) \quad ; \quad f_{n}=f(x+n h)
$$

The difficulty is to choose a value of $h$ which is not too small (otherwise significant figures will be lost in evaluating the numerators of eqs (28)-(30)) but not too large (otherwise the truncation error, which can be estimated by the second term of eqs (28)-(30), will be large). One also has to consider the word length of the computer and the convenience of using single versus double precision. In this work we calculated on a CDC 6400 and a CDC 6600 machine with a 60 bit word length (13 significant figures).

We were able to observe definitely the effect of varying $h$ for the special case $\theta=\phi=1$, i.e., for classical corresponding states. Equations (19) and (20) have been solved analytically by Wielopolski (1980) and the lengthy expressions are reported in an NBS publication [1] and will not be repeated here. For example, table 2 lists the number of figures in the numerical results which were in agreement with the analytical results for the first, second, and third derivatives of $\widetilde{G}_{x} / R T$ for a particular test case. The number of figures in agreement for the function value of $\widetilde{G}_{x} / R T$ itself was $10-12$.

TABLE 2. Comparison of Numerical and Analytical Results for Derivative Calculations Using Single Precision Arithmetic. Subscripts refer to the derivation with respect to $x$.

\begin{tabular}{cccc}
\hline \hline$h$ & $\left(\tilde{G}_{x} / R T\right)_{x}$ & $\left(\bar{G}_{x} / R T_{2 x}\right.$ & $\left(\tilde{G}_{x} / R T\right)_{3 x}$ \\
\hline $10^{-3}$ & 6 & 5 & 4 \\
$10^{-3}$ & 7 & 5 & 3 \\
$10^{-3}$ & 7 & 3 & 0 \\
\hline
\end{tabular}

Table 2 indicates that the first order derivative is truncation error controlled, since its value becomes more accurate as $h$ is decreased. The second and third derivatives are, on the other hand, controlled by the loss of significant figures since as $h$ is decreased, they lose accuracy. Since the third order derivative is the least accurate, we chose the value of $h$ for which it is calculated most accurately.

We now consider what the smallest values of the second and third order derivatives are which can be calculated with $h=10^{-3}$, since our eventual goal is to solve the equations for the critical point by driving the values of those derivatives to zero. The derivatives go to zero by a cancellation of the two terms in eqs (20) and (21), that is, the contribution from the hypothetical substance is cancelled by the ideal mixture contribution. For this reason, the values of the derivatives cannot be made arbitrarily small. The ideal mixture contribution (which can be computed with negligible error) can only cancel as many significant figures as appear in the hypothetical substance contribution. Consider the case in table 2 with $h=10^{-3}$. For the second order derivative, the hypothetical substance contribution has five 
significant figures, and its value is order unity (abbreviated $0(1)$ ). If the ideal mixture contribution were to cancel all five of these figures, the result would be a number of $0\left(10^{-5}\right)$ with no significant figures remaining. For the third order derivative, the hypothetical substance contribution contains four significant figures, and is $O(10)$. Cancelling all significant figures would leave a number of $O\left(10^{-3}\right)$.

In our first attempt at calculating critical lines based on the numerical evaluation of the derivatives in eqs (20) and (21) using single precision arithmetic, we were unable to obtain convergence of the temperature and pressure to five significant figures. The problem appeared to be that there were not enough significant figures in the derivative calculations. While the truncation error is inherent to the formulas being used, the loss of significant figures can be compensated by adding more figures to the function values. This was done by the use of double precision arithmetic which gives us 26 significant figures on the CDC 6400. In table 3 below, the results for the numerical derivatives calculated using double precision arithmetic are compared with the results arrived at analytically. Again, reported in the table are the number of figures of agreement between the two results.

TABLE 3. Comparison of Numerical and Analytical Results for Derivative Calculations Using Double Precision Arithmetic.

\begin{tabular}{lccc}
\hline \hline$h$ & $\left(\tilde{G}_{x} / R T\right)_{x}$ & $\left(\tilde{G}_{x} / R T\right)_{2 x}$ & $\left(\widetilde{G}_{x} / R T\right)_{3 x}$ \\
\hline $10^{-3}$ & 6 & 5 & 4 \\
$10^{-4}$ & 7 & 9 & 6 \\
$10^{-5}$ & 7 & 8 & 7 \\
$10^{-6}$ & 7 & 8 & 7 \\
$10^{-7}$ & 7 & 8 & 7 \\
$10^{-8}$ & 7 & 8 & 4 \\
$10^{-9}$ & 7 & 8 & 1 \\
\hline
\end{tabular}

For $h=10^{-3}$, the single and double precision results are the same, which indicates that truncation error is control. ling. Looking at the double precision results, the third derivative shows an increase in accuracy as $h$ is decreased to $10^{-5}$; clearly indicating that the truncation error is decreasing to this point. As $h$ is decreased past $10^{-7}$, accuracy is lost due to loss of significant figures.

Based on these results, a value of $h=10^{-6}$ is chosen to compute the derivatives in double precision. Given this value for $h$, the smallest value of the second derivative which may be calculated (containing no significant figures) is $O\left(10^{-8}\right)$, and that for the third derivative is $O\left(10^{-6}\right)$. The calculations of the binary critical line were subsequently made to converge to five significant figures for both temperature and pressure.

\section{Results}

It must again be stressed that the general procedure for calculating the critical line or VLE is predictive and requires only the critical constants and an acentric factor for the fluid of interest, or of the components in a mixture. For a relatively simple system the results will be reasonable without optimization of any parameters. Since, however, we are concerned only with VLE and the critical point we considered two straightforward optimization procedures involving the factor $\omega$. The first was to adjust $\omega$ to give the best representation of the pure component vapor pressure curve, the second was to force the critical temperature and pressure of the pure fluids to correspond exactly with those of the reference substance. This second variation is simply to set $\omega_{\alpha}=\omega_{0}$ : hence by eqs (24) and (25) $\theta=1$ and $\phi=$ $Z_{o}^{c} / Z_{\alpha-a}^{c}-a$ form of classical corresponding states. One should note that the two procedures are not the same because the Leach-Leland equations are not constrained at the critical point.

\section{Ethane: Pure Component Results:}

We first considered the ethane vapor pressure curve which was obtained using Leach's expression for the shape factors. The value of the acentric factor for the Leach equations was determined by optimizing agreement with the vapor pressure data by a trial and error procedure in which the sum of the average absolute deviations, for the vapor pressure, and saturated vapor and liquid densities, were minimized; temperature being chosen as the independent variable. The temperature range over which the results were optimized was $180 \mathrm{~K}$ to $300 \mathrm{~K} ; 180 \mathrm{~K}$ being the lower limit for which Leach's equations were designed; $300 \mathrm{~K}$ corresponded to the maximum temperature for which the vapor pressure program would converge. Calculated and data values were compared at $10 \mathrm{~K}$ increments. The average deviations obtained for several values of acentric factor are given in table 4. The value chosen for acentric factor in this work $(\omega=0.094)$ is seen to give a substantial improvement over the Pitzer value $(\omega=0.105)$ which was used by Leach.

TABLE 4. Variation of Ethane Vapor Pressure Curve With Acentric Factor (Leach $\theta, \phi)$.

\begin{tabular}{|c|c|c|c|c|}
\hline $\begin{array}{l}\text { Acentric } \\
\text { Factor } \omega\end{array}$ & $\begin{array}{c}\text { Ave } \% \\
\frac{|\Delta p|}{p}\end{array}$ & $\begin{array}{c}\text { Ave } \% \\
\left|\Delta_{Q_{\text {rap }}}\right|\end{array}$ & $\begin{array}{c}\text { Ave } \% \\
\left|\Delta Q_{u_{q}}\right| \\
Q_{u_{q}}\end{array}$ & $\Sigma$ Ave $\%$ \\
\hline 0.105 & 2.080 & 3.148 & 0.6158 & 5.844 \\
\hline .100 & 1.259 & 2.215 & .3706 & 3.845 \\
\hline .096 & 0.595 & 1.460 & .3791 & 2.434 \\
\hline .095 & .467 & 1.270 & .3894 & 2.126 \\
\hline .094 & .444 & 1.164 & .4067 & 2.015 \\
\hline .093 & .479 & 1.127 & .4245 & 2.031 \\
\hline .092 & .543 & 1.137 & .4424 & 2.122 \\
\hline .090 & .736 & 1.241 & .4904 & 2.467 \\
\hline .005 & 60.579 & 61.633 & 4.746 & 126.958 \\
\hline
\end{tabular}


The curves for vapor pressure and orthobaric densities $(\varrho=1 / V)$, obtained using Leach's shape factors with the optimized acentric factor are compared with the correlations of Goodwin, et al. [15] to obtain the deviations plotted in figure 1.

Also in figure 1, deviations for the vapor pressure and orthobaric density curves predicted using classical corresponding states are presented. This figure emphasizes that the Leach shape factors make a significant difference. The vapor pressure deviations are positive, and become larger as the triple point is approached, since classical corresponding states predicts a slope of the vapor pressure curve $(d P / d T)$ which is too small. The deviation of pressure goes to zero at the critical point because the two parameters are choosen to make the critical temperature and pressure correspond exactly.

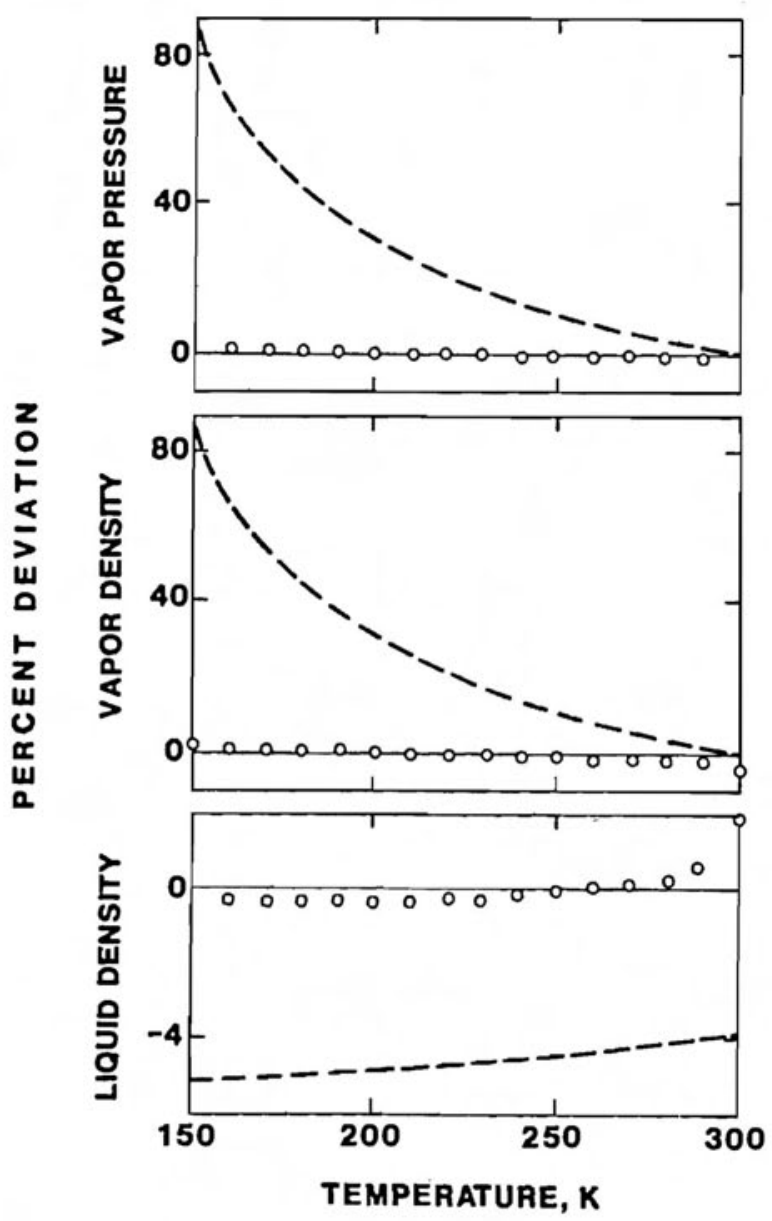

Figure 1. Percent deviations [(expl-calc) $\times 100 /$ calc] for the vapor pressure, vapor density and liquid density for ethane at saturation. Data from Goodwin [15] Calculations from the extended corresponding states method with the parameter $\omega_{0}$ optimized, circles. Also shown as the dashed line are the results with $\theta=1$ and $\phi=Z_{i} / Z_{s}^{s}$, see equations (22)-(27).
The critical point results for ethane are in table 5 . Notice that the results are better using classical corresponding states than with the Leach shape factors. This is because classical corresponding states forces either the critical temperature and density to correspond $(\theta=\phi=1)$, or it forces the critical temperature and pressure to correspond $\left(\theta=1, \phi=Z_{o}^{c} / Z_{\alpha}^{c}\right)$.

TABle 5. Ethane Critical Point Predictions.

\begin{tabular}{lcccc}
\hline & \multirow{2}{*}{ Data } & $\begin{array}{c}\text { Leach } \theta, \\
\phi(\omega=0.094)\end{array}$ & $\theta=\phi=1$ & $\begin{array}{l}\theta=1, \\
\phi=Z_{\delta} / Z_{\mathrm{f}}\end{array}$ \\
\hline$T_{c}(\mathrm{~K})$ & 305.33 & $307.01(0.55)^{\circ}$ & 305.33 & 305.33 \\
$P_{c}(\mathrm{bar})$ & 47.488 & $48.790(2.83)$ & $47.750(0.55)$ & 47.448 \\
$\varrho_{c}(\mathrm{~mol} / \mathrm{L})$ & 6.80 & $6.98(2.65)$ & 6.80 & $6.76(-.59)$ \\
\hline
\end{tabular}

- Percent deviation is in parentheses.

\section{The Critical Line:}

We first calculate the critical line using the Leach shape factor equations with acentric factors of 0.005 and 0.094 for methane and ethane respectively. The results are plotted against the critical line data found in the review article of Hicks and Young [16], and identified in the caption to figure 2 (the symbols used in figure 2 are identical with those used in figures 3 through 7).

The results are presented in the form of $T-x$ and $p-x$ plots in figures 2-5 and show the general trends obtained by varying the binary interaction parameters, $\xi$ and $\eta$. Holding $\xi$ constant, figures 2 and 3 show that $\eta$ has a small effect on the $T \cdot x$ curve, and a large effect on the $p-x$ curve. In both cases, increasing $\eta$ gives a better representation of the data.

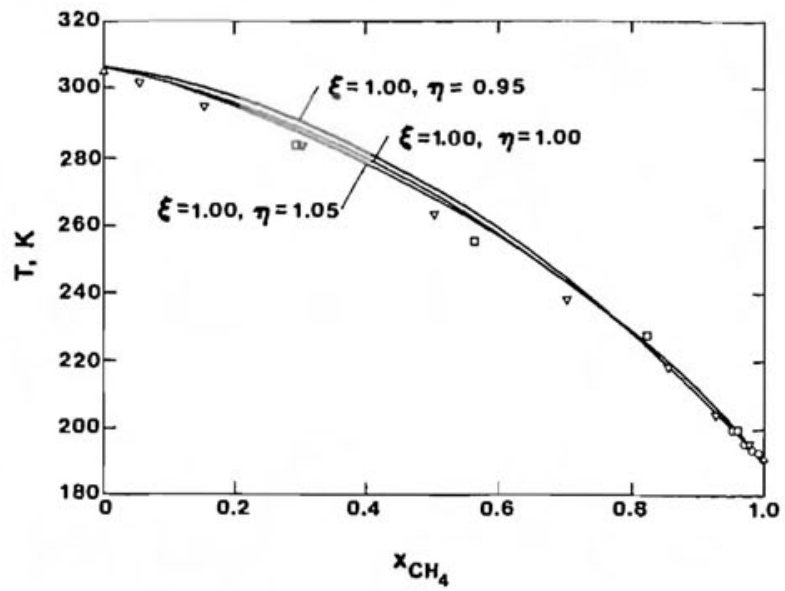

Ficure 2. The methane-ethane $T-x$ critical plot calculated via extended corresponding states showing the variation caused by the interaction parameter, $\eta$. Data [16]: $\nabla$ Bloomer, Gani and Parent; $\square$ Price and Kobayashi; $O$ Wicheterle and Kobayashi. The values for the pures, $\Delta$, are from Goodwin [15] and [19]. 
Holding $\eta$ constant, figures 4 and 5 show that $\xi$ has a much larger effect on the $T-x$ curve than did $\eta$, and an equally large effect on the $p$ - $x$ curve. The important point to notice is that the maximum value in the $p-x$ curve is shifted towards small mole fraction values (of $\mathrm{CH}_{4}$ ) by decreasing $\xi$. The best representation of the $P$ - $x$ curve in figure 3 (i.e., $\xi=1.00, \eta=1.08$ ) indicates that the peak of the curve needs to be shifted towards the smaller mole fractions to improve the agreement, thus, $\xi$ should be decreased.

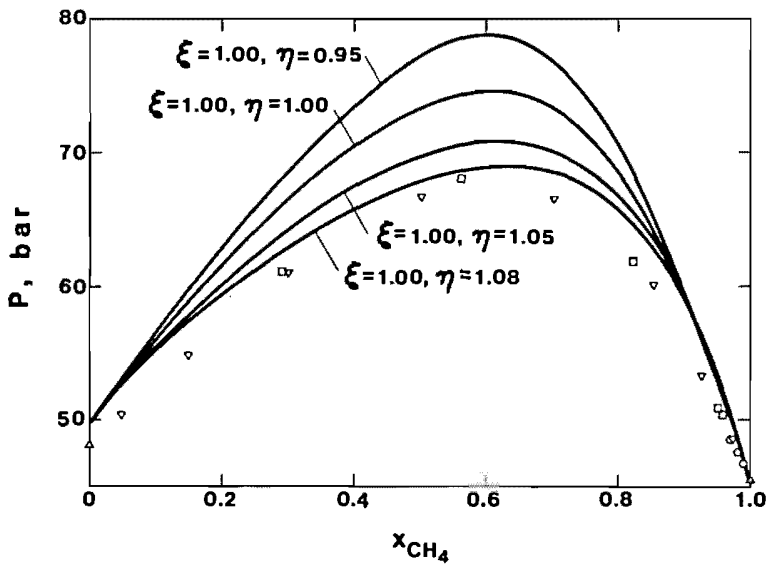

Figure 3. The p-plot corresponding to figure 2.

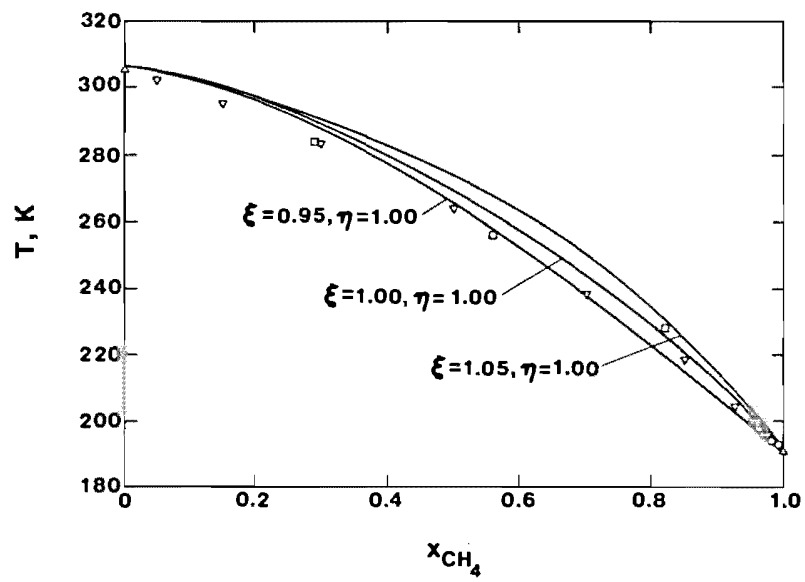

Figure 4. Variation of the parameter, $\xi$, for methane-ethane in the $T-x$ plot.

To achieve the goal of obtaining the interaction parameters by fitting the critical line data, a manual search technique was initiated. The "best fit" was defined in the least squares sense. The results of this search were that $\xi=0.97$, and $\eta=1.13$ were chosen as the "best" values for the interaction parameters. The "best fit" $T-x$ and $p-x$ curves are presented in figures 6 and 7 respectively.

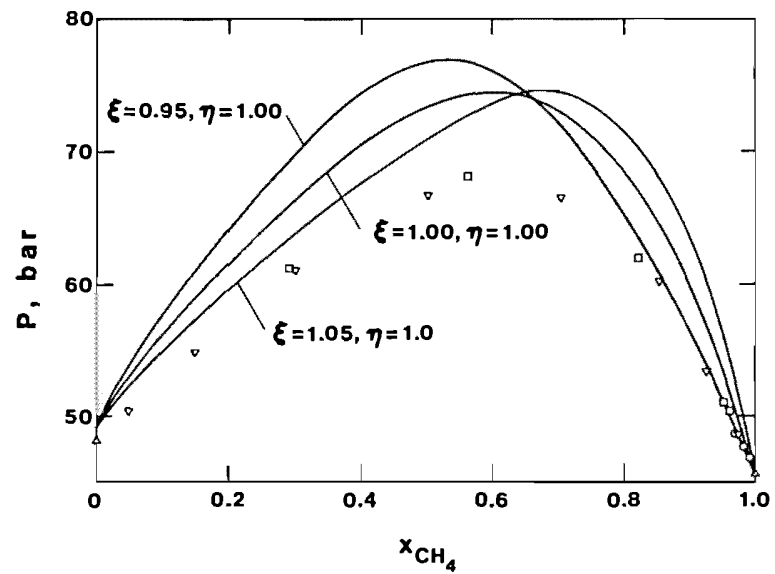

FIGURE 5. The p-x plot corresponding to figure 4.

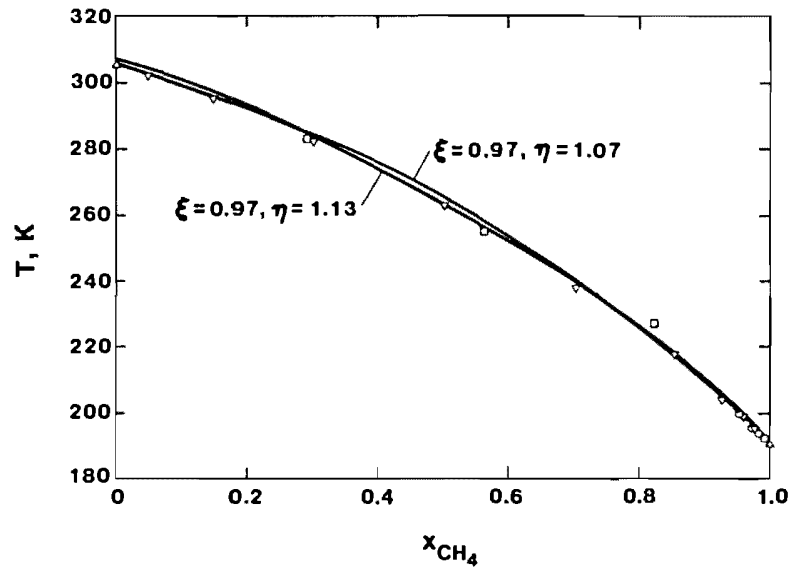

Ficure 6. The $T$ - $x$ critical line of methane-ethane with optimized interaction parameters. The two curves correspond to extended corresponding states $[\xi=0.97, \eta=1.13]$ and the classical corresponding states with $\omega_{a}$ $=\omega_{0}[\xi=0.97, \eta=1.07]$.

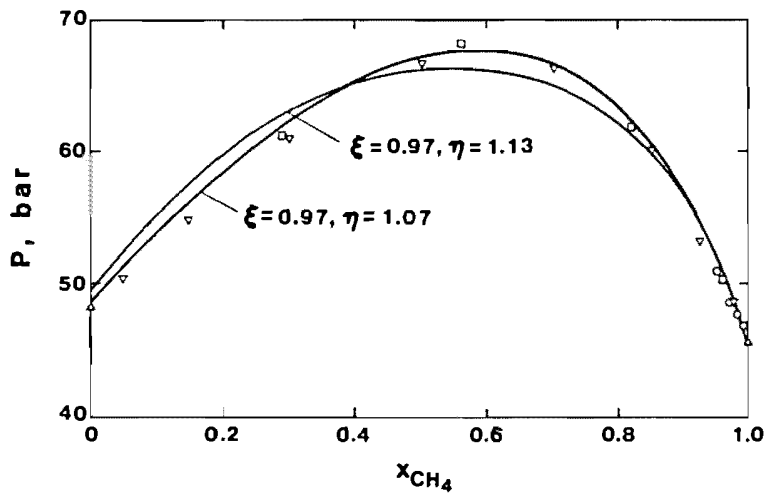

Figure 7. The $p-x$ plot corresponding to figure 6 . 
The fit of the $T-x$ curve is good, with only one data point which seems astray. The $p-x$ curve, however, does not have the right shape to fit the data well. Part of the fitting problem is due to the bad prediction which is made for the critical point of pure ethane. This led us to try the second approach of setting $\omega_{a}=\omega_{0}$. Hence, the critical endpoints in the $T-x$, and $p-x$ curves are exact. A new optimization led to the parameter values $\xi=0.97, \eta=1.07$. While the fit of the $T$ - $x$ curve was not significantly improved that for the $p-x$ curve was. These results are shown in figures 6 and 7 .

\section{Vapor-Liquid Equilibria Results:}

Of the VLE data judged to be thermodynamically consistent by Hiza, et al. [2], three representative isotherms were chosen to test the predictions made using the binary interaction parameters determined in the previous section. Two of the isotherms are supercritical ( $250 \mathrm{~K}$ and $199.92 \mathrm{~K}$ ), and

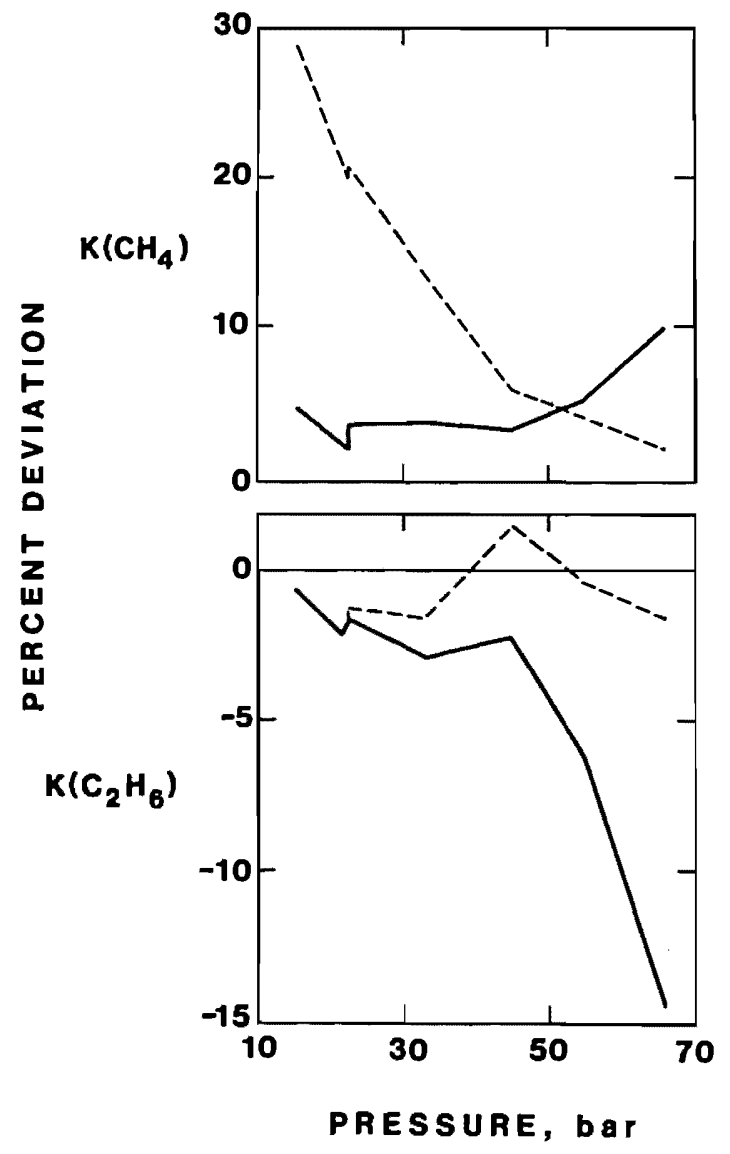

Figure 8. Methane thane $K$-value deviation plots at $250 \mathrm{~K}$. Shown are the (dashed) curves with the interaction parameters from the critical line fit $[\xi=0.97, \eta=1.07]$ and, for reference, with $\xi=\eta=1.00$ as the solid curves. one is subcritical $(144.26 \mathrm{~K})$. The sources of the data are: $250 \mathrm{~K}$ isotherm, Davalos, et al. [17]; $199.92 \mathrm{~K}$ and $144.26 \mathrm{~K}$ isotherms, Wichterle and Kobayashi [18].

The VLE calculations used the Leach shape factors with the acentric factors 0.005 and 0.094 for methane and ethane respectively. The results are presented as $K$-value deviation plots for both the methane and the ethane $K$-value predictions. Figures 8, 9, and 10 contain these curves with the interaction parameters obtained from the critical line fit (i.e., $\xi=0.97, \eta=1.07$ ). These figures also show that setting the interaction parameters to unity gives much better VLE predictions than do the parameters obtained from the best fit of the critical line data.

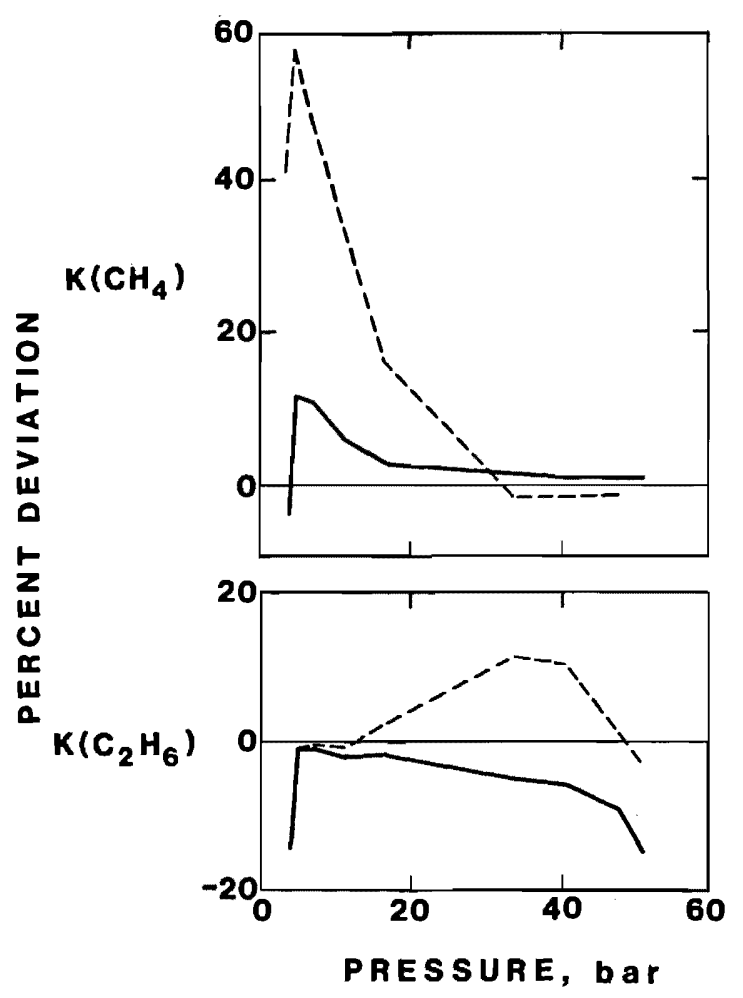

FIGURE 9. Methane-ethane $K$-values at $199.92 \mathrm{~K}$.

\section{Summary and Conclusions}

The proposed technique of calculating binary critical lines by numerically evaluating the second and third order derivatives of the Gibbs free energy has been checked with an analytical solution for the special case of classical corresponding states, and has proven successful. The best least squares fit of the critical line data of the system methaneethane was then shown to be poor (particularly the $p-x$ curve) if the Leach shape factors are used with an acentric 
factor optimized for pure component vapor pressure predictions. This is due to a bad prediction of the critical endpoint for ethane. To improve this fit, we use classical corresponding states to force correspondence of the temperature and pressure at the critical line endpoints. However we also show that the pure component vapor pressure predictions are not satisfactory if this is done. Finally, VLE predictions are made using Leach shape factors with the acentric factor optimized for vapor pressure predictions, and the binary interaction parameters obtained from the best fit of the critical line data (i.e., with $\theta=1, \phi=Z_{o}^{c} / Z_{c}^{c}$ ). The results are not as good in general as those which are obtained by setting $\xi=\eta=1$. Hence we conclude that a fit of the binary critical line does not yield binary interaction parameters of any general significance.

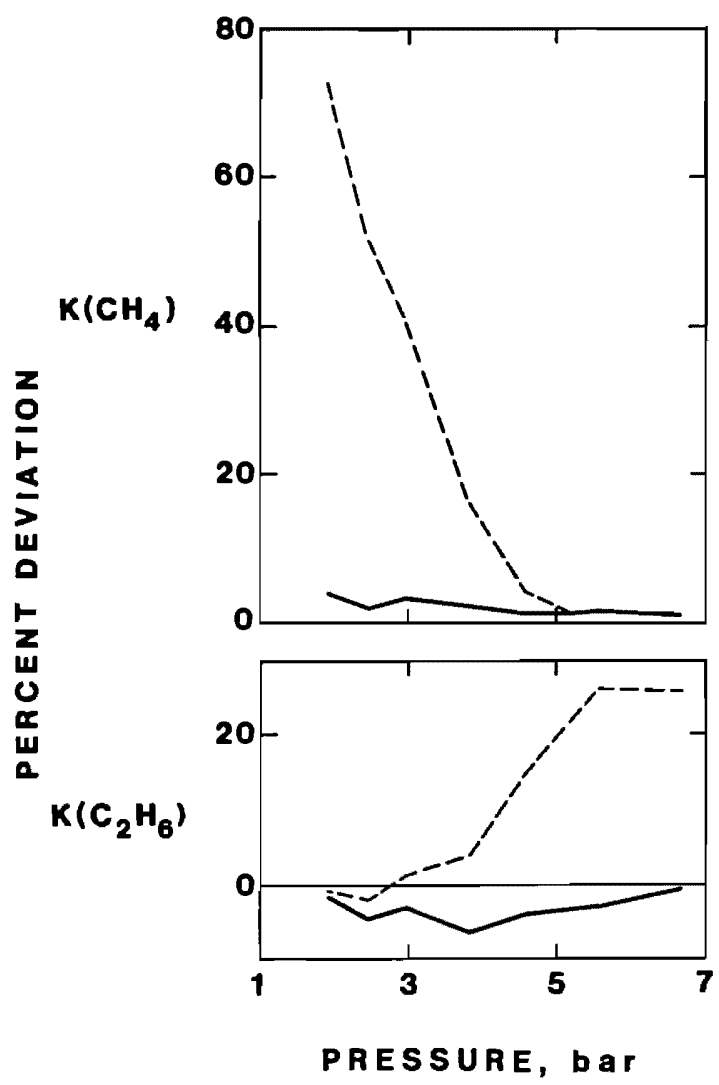

FICURE 10. Methane-ethane $K$-values at $144.26 \mathrm{~K}$.

\section{References}

[1] Wielopolski, P. On the calculation of critical liquid-vapor lines of binary mixtures. J. Res. Nat. Bur. Stand. (U.S.) 85(6): 441-449; 1980 November-December.

[2] Hiza, M. J.; Miller, R. C.; Kidnay, A. J. A review, evaluation, and correlation of the phase equilibria, heat of mixing and change in volume on mixing for liquid mixtures of methane plus ethane. J. Phys. Chem. Ref. Data 8(3): 799-816; 1979.

[3] Watson, I. D.; Rowlinson, J. S. The prediction of the thermodynamic properties of fluids and fluid mixtures. II. Liquid-vapor equilibrium in the system argon + nitrogen + oxygen. Chem. Eng. Sci. 24: 1575-1580; 1969.

[4] Gunning, A. J.; Rowlinson, J. S. The prediction of the thermodynamic properties of fluids and fluid mixtures. III. Applications. Chem. Eng. Sci. 28: 521-527; 1973.

[5] Teja, A. S.; Rowlinson, J. S. The prediction of the thermodynamic properties of fluids and fluid mixtures. IV. Critical and azeotropic states. Chem. Eng. Sci. 28: 529-538; 1974.

[6] Mollerup, J.; Rowlinson, J. S. The prediction of the densities of liquefied natural gas and of lower molecular weight hydrocarbons. Chem. Eng. Sci. 29: 1373-1381; 1974.

[7] Mollerup, J. Correlated and predicted thermodynamic properties of LNG and related mixtures in the normal and critical regions. Paper E-2 in Advances in Cryogenic Engineering, Vol. 20. K. D. Timmerhaus, ed. New York, NY: Plenum Publishing Corp.; 1975. 172-194.

[8] Mollerup, J. Thermodynamic properties of natural gas, petroleum gas, and related mixtures: enthalpy predictions. Paper $M-1$ in Advances in Cryogenic Engineering, Vol. 23. K. D. Timmerhaus, ed. New York, NY: Plenum Publishing Corp.; 1978. 550-560.

[9] Henderson, D.; Leonard, P. J. Physical Chemistry. Edit Eyning, H.; Henderson, D.; Jost, W.; (Academic Press, New York, 1971) Chapter 7, "Liquid Mixtures."

[10] Eaton, B. E. Prediction of the critical line of binary mixtures: Determination of binary interaction parameters. M.S. Thesis, University of Colorado, 1980. $151 \mathrm{p}$.

[11] Leach, J. W.; Chappelear, P. S.; Leland, T. W. Use of molecular shape factors in vapor-liquid equilibrium calculations with the corresponding states principle. A.I.Ch.E. J. 14(4): 568-576; 1968.

[12] Ely, J. F.; Hanley, H. J. M. Ind. and Eng. Chem. Fund. (in press, 1981).

[13] McCarty, R. D. A modified Benedict-Webb-Rubin equation of state for methane using recent experimental data. Cryogenics 14(5): 276-280; 1974.

[14] Hildebrand, F. B. Introduction to Numerical Analysis, 2nd ed. New York, NY: McGraw-Hill; 1974. 669 p.

[15] Goodwin, R. D.; Roder, H. M.; Straty, G. C. Thermophysical properties of ethane, from 90 to $600 \mathrm{~K}$ at pressures to $700 \mathrm{bar}$. Nat. Bur Stand. (U.S.) Tech. Note 684; 1976 August. 320 p.

[16] Hicks, C. P.; Young, C. L. The gas-liquid critical properties of binary mixtures. Chemical Reviews 75(2): 139-175; 1975 April.

[17] Davalos, J.; Anderson, W. R.; Phelps, R. E.; Kidnay, A. J. Liquid-vapor equilibria at $250.00 \mathrm{~K}$ for systems containing methane, ethane, and carbon dioxide. J. Chem. Eng. Data 21(1): 81-84; 1976.

[18] Wichterle, 1.; Kobayashi, R. Vapor-liquid equilibrium of methaneethane system at low temperatures and high pressures. J. Chem. Eng. Data 17(1): 9-12; 1972.

[19] Goodwin, R. D. The thermophysical properties of methane, from 90 to $500 \mathrm{~K}$ at pressures to 700 bar. Nat. Bur. Stand. (U.S.) Tech. Note 653; 1974 April. 274 p. 